\title{
Electrophysiological Monitoring of Neurochemical-based Neural Signal Transmission in a Human Brain-Spinal Cord Assembloid
}

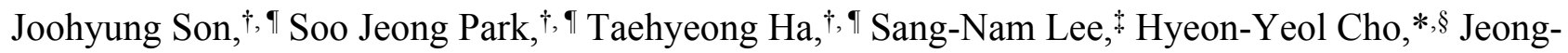
Woo Choi*,

† Department of Chemical \& Biomolecular Engineering, Sogang University, Seoul, 04107, Republic of Korea

₹ Uniance Gene Inc., 1107 Teilhard Hall, 35 Baekbeom-Ro, Mapo-Gu, Seoul, 04107, Republic of Korea

$\S$ Department of Bio \& Fermentation Convergence Technology, Kookmin University, Seoul, 02707, Republic of Korea

I Equal contribution

*Corresponding authors: jwchoi@sogang.ac.kr (J.-W.C.), chohy@kookmin.ac.kr (H.-Y.C.)

\section{TABLE OF CONTENTS}

Figure S1. Generation of cerebral organoids.

Figure S2. Immunofluorescence analysis for representative marker expression of the CO.

Figure S3. Characterization of generated motor neuron spheroids (MNSs).

Figure S4. The process of fabrication of a 3D-engineered chip and eBSA.

Figure S5. Confirmation of the connection between CO and MNS of eBSA.

Figure S6. Comparison of electrophysiological activity with normalized spike number.

Figure S7. Electrophysiological analysis of eBSA consisted with SH-SY5Y spheroids.

Figure S8. Evaluation of glutamate-mediated excitatory effects of caffeine in CO.

S-6

Supplementary Table 1. List primers for RT-PCR. 


\section{Supplementary Figures}

a

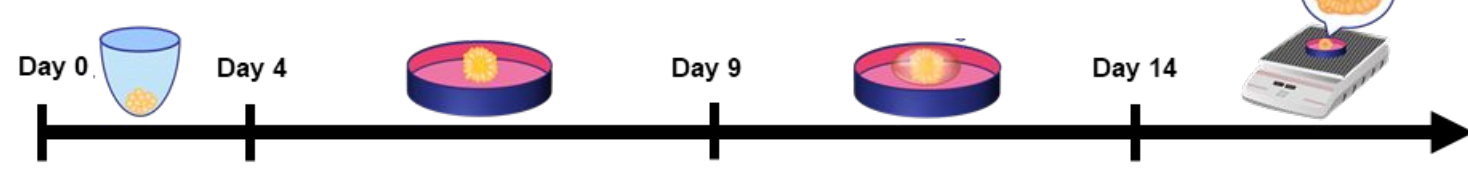

Generation of Germ layer differentiation and Expansion of neuroepithelial embryoid body induction of primitive neuroepithelia tissue with Matrigel embedding

Growth of cerebral tissue

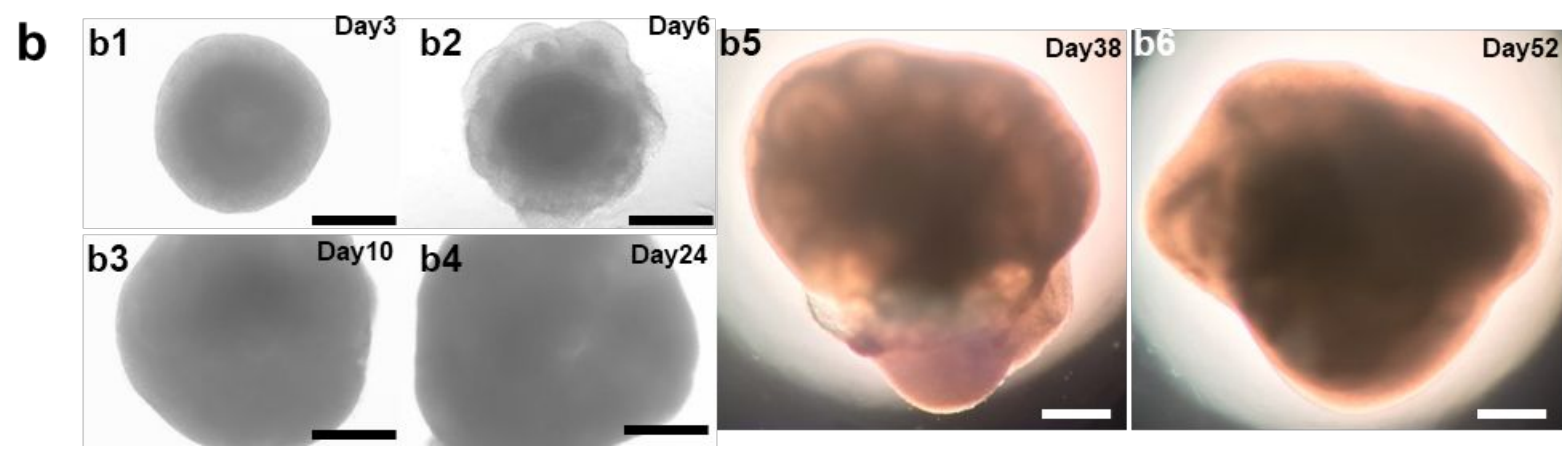

Figure S1. Generation of cerebral organoids. (a) Graphical protocol for a cerebral organoid generation. (b) Time-dependent morphological change of $\mathrm{CO}$ (b1: day3, b2: day6, b3: day10, b4: day24, b5: day38, b6: day52, phase image), scale bar: $500 \mu \mathrm{m}$.
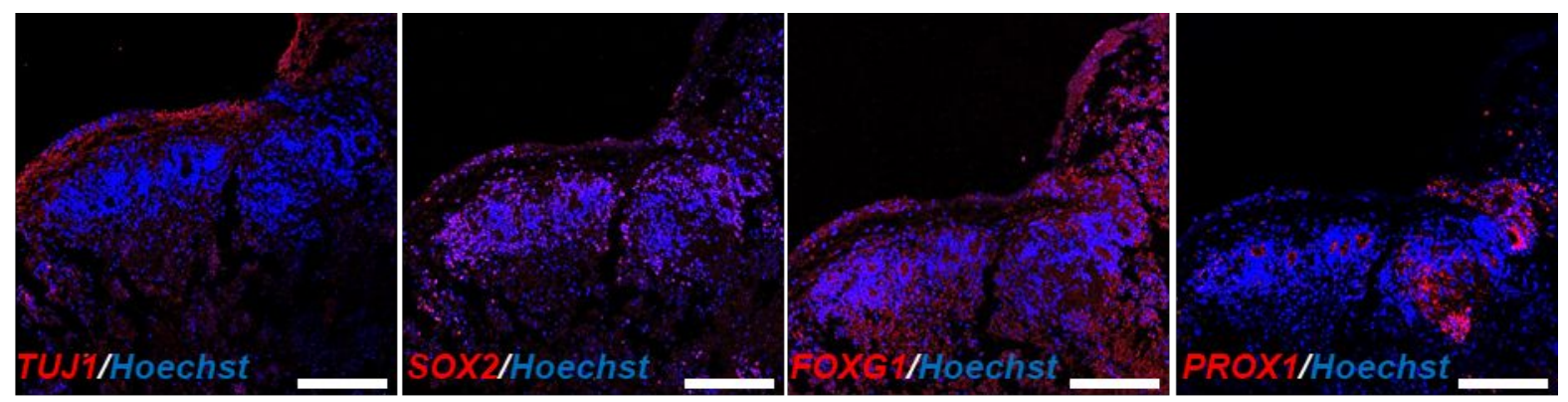

Figure S2. Immunofluorescence analysis for representative marker expression of the CO. Blue: Hoechst, scale bar: $100 \mu \mathrm{m}$. 
a

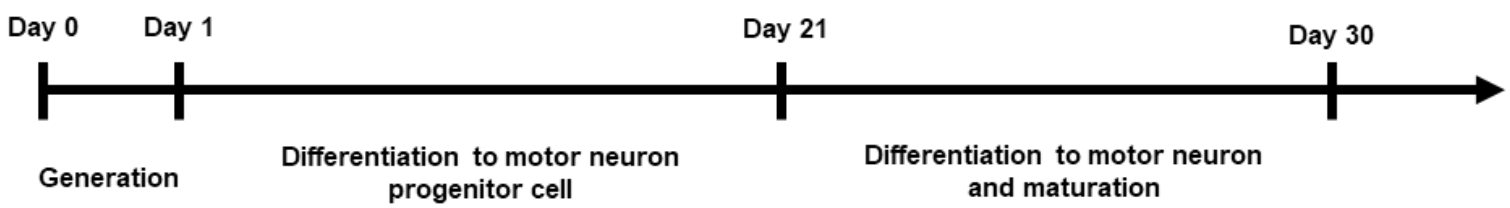

b
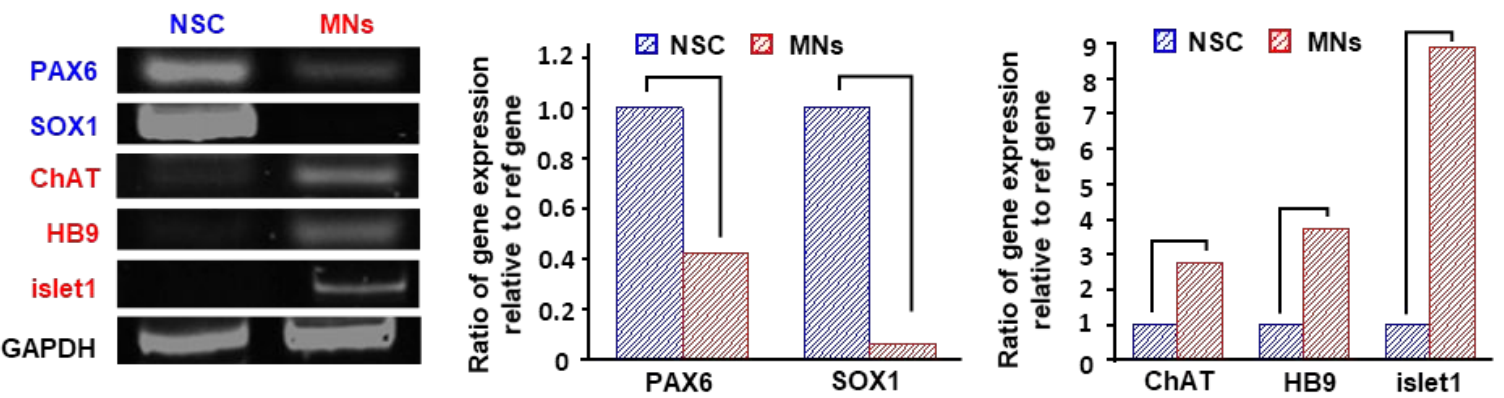

Figure S3. Characterization of generated motor neuron spheroid (MNS). (a) Timeline of the formation process of MNSs, (b) mRNA expression levels of MNSs' representative markers.
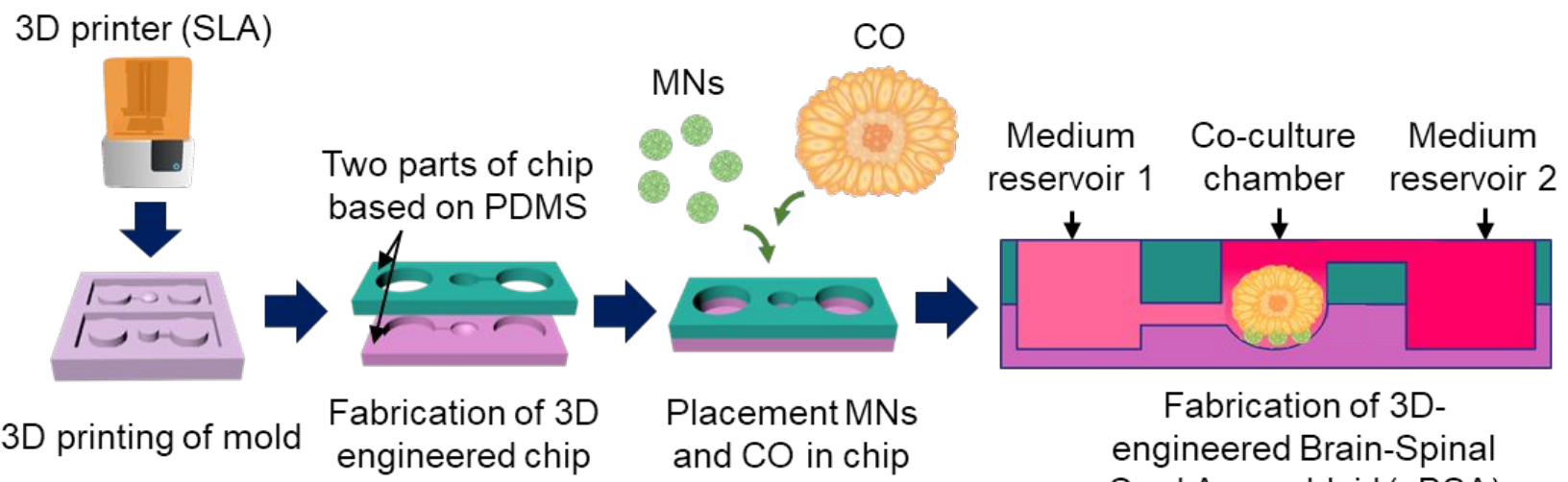

Fabrication of 3D-

engineered Brain-Spinal

Cord Assembloid (eBSA)

Figure S4. The process of fabrication of a 3D-engineered chip and eBSA. 


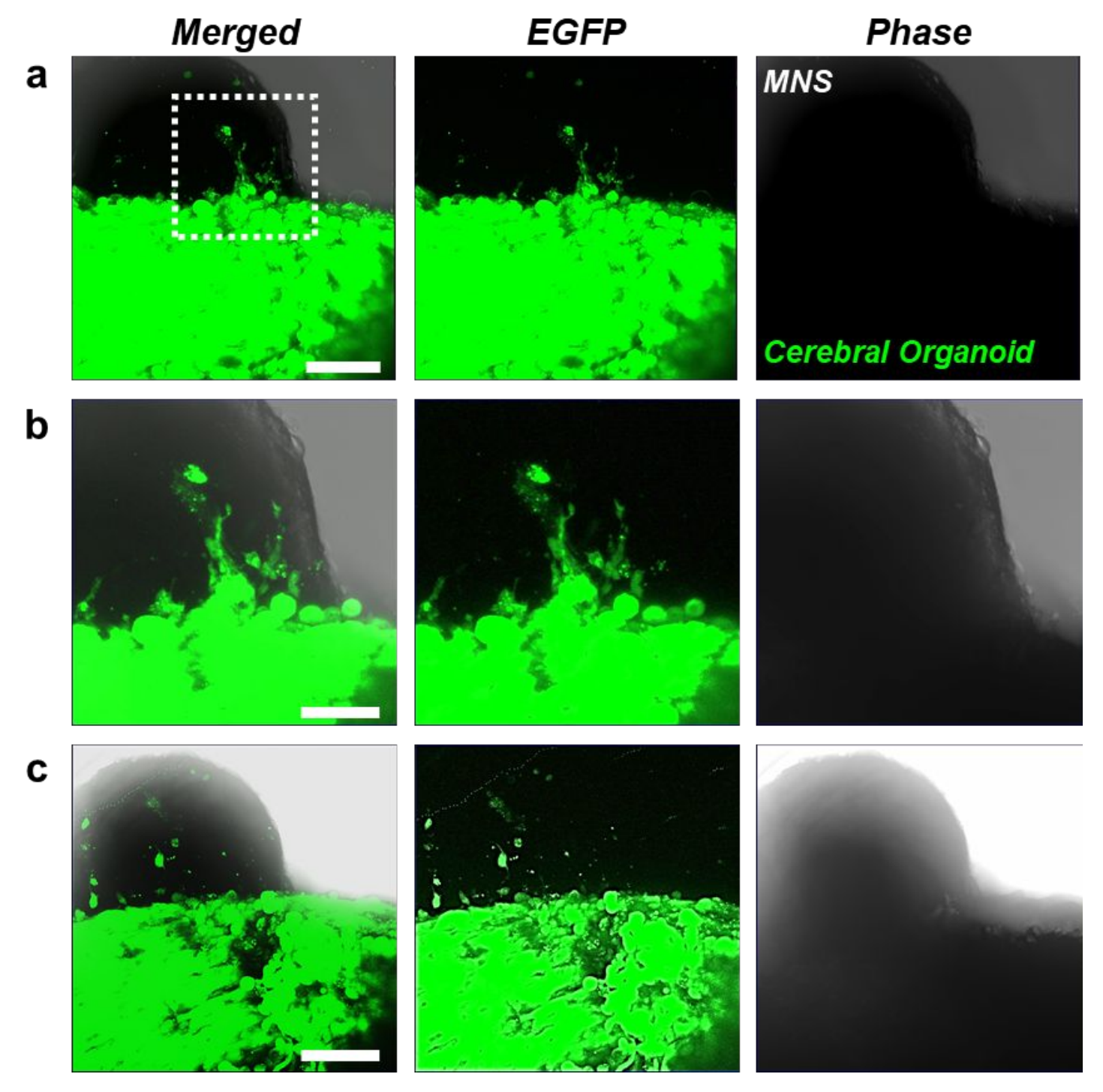

Figure S5. Confirmation of the connection between cerebral organoid (CO) and motor neuron spheroid (MNS) of eBSA. eBSA was assembled with EGFP-labeled CO and MNS, and its connection was monitored at day 3 and day 7 by confocal fluorescence microscopy. Microscopic image of (a) day 3 from assembly, (b) Zoomed-in image of (a), (c) day 5 from assembly. Scale bar: (a) $100 \mu \mathrm{m}$, (b) $50 \mu \mathrm{m}$, (c) $100 \mu \mathrm{m}$. 


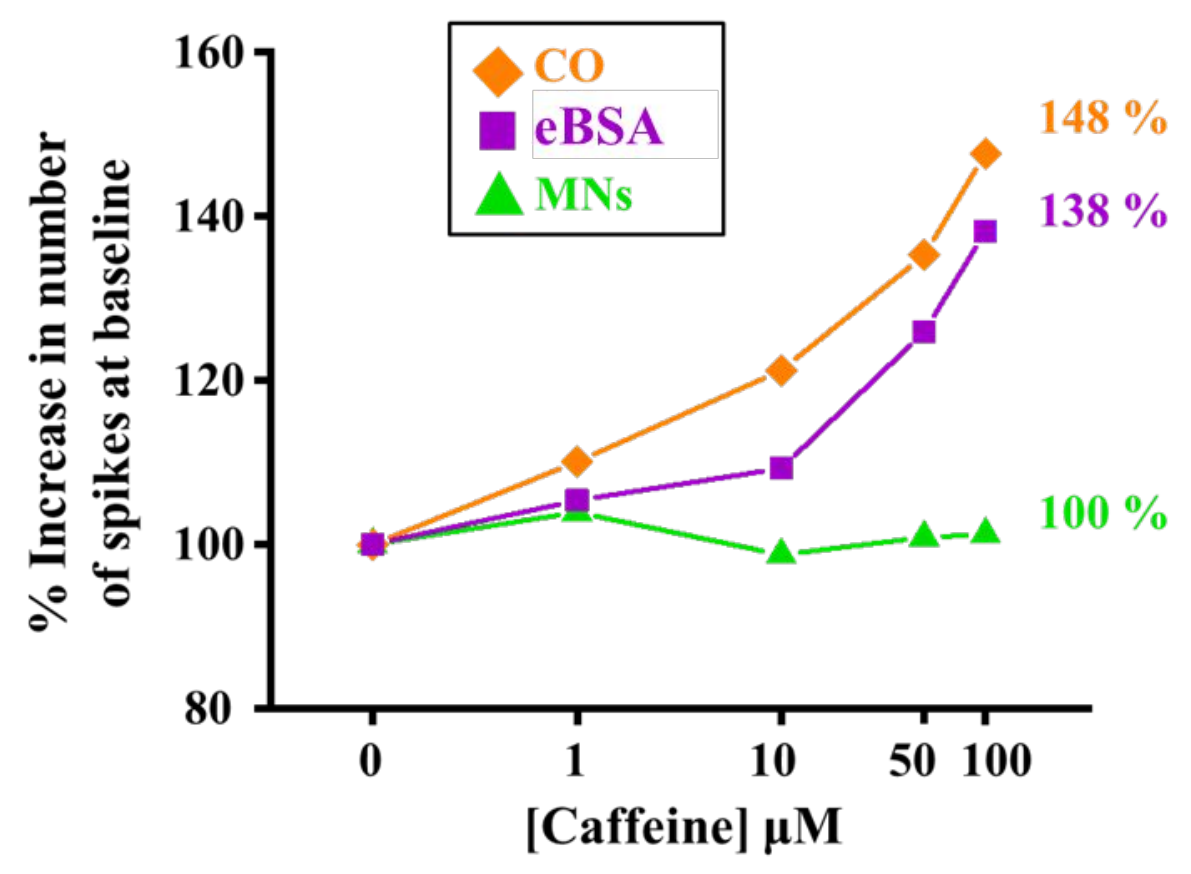

Figure S6. Comparison of electrophysiological activity with normalized spike number.

a

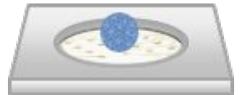

SH-SY5Y spheroid (neuroblastoma)

b

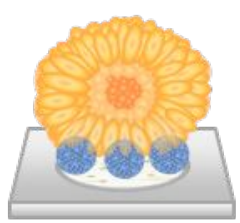

CO fused with SH-SY5Y spheroids
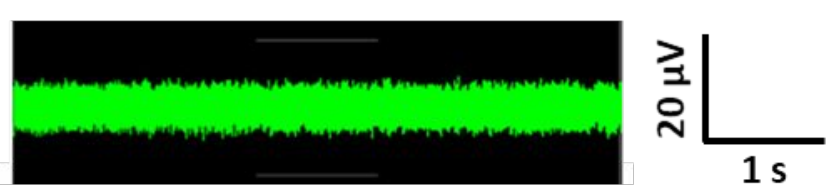

Burst Frequency

$50 \mathrm{sec}$
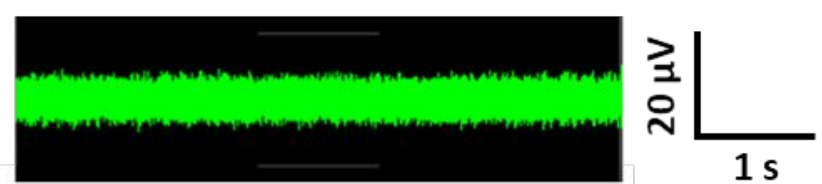

Burst Frequency

$$
50 \mathrm{sec}
$$

Figure S7. Electrophysiological analysis of eBSA consisted with SH-SY5Y spheroids. Spontaneous electrophysiological activity analysis with $100 \mu \mathrm{M}$ caffeine treatment on (a) SH-SY5Y spheroids and (b) eBSA with SH-SY5Y spheroids. 


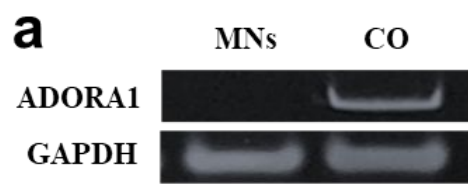

b

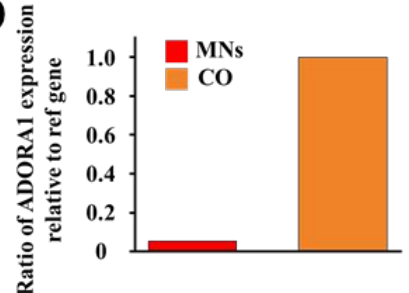

C

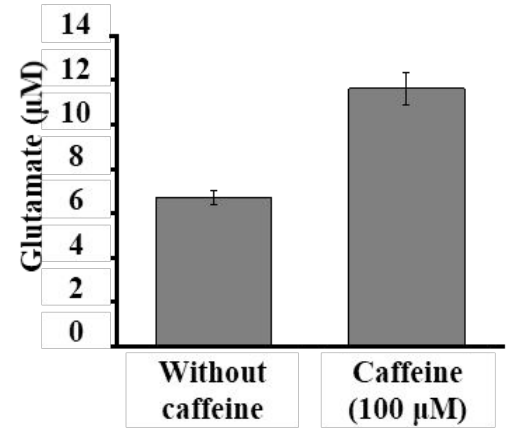

d

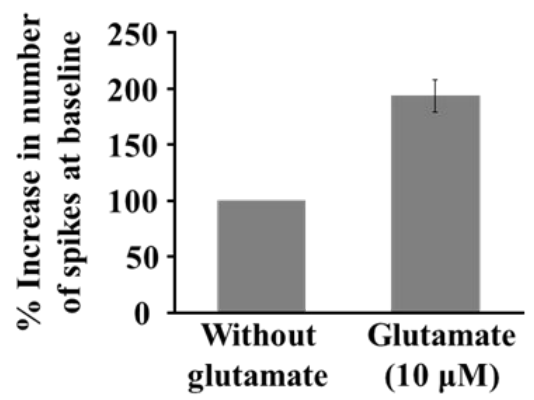

Figure S8. Evaluation of glutamate-mediated excitatory effects of caffeine in CO. (a) mRNA expression analysis of the adenosine receptor 1 related gene (ADORA1) in MNSs and CO and (b) its histogram. (c) The glutamate release profile of CO before and after the caffeine treatment. (d) Electrophysiological analysis of MNSs with glutamate treatment.

Supplementary Table 1. List primers for RT-PCR.

\begin{tabular}{|c|c|c|}
\hline Gene & Forward Primers & Reverse Primers \\
\hline PAX6 & AGCGGCTCTAACAGCCATTT & AGACGAAGCTCAGTAGGGGG \\
\hline SOX1 & GTCCAATGGGACCTTGAGGG & AGCACCTGGCATTGGCTATT \\
\hline ChAT & CCCACTAGCTGGAATCAGGC & GTACTGCCCATCCAGCCTTT \\
\hline HB9 & TTGGAGGGGCATTCGTTACC & TGAGACGGGCAGCGAATTAT \\
\hline islet1 & TTGCTTAGCCTGTGCAGACA & TCCATCTGGGAGCTGACACT \\
\hline ADORA1 & ACATTGGGCCACAGACCTAC & TGGCCCAAAGAACATCTGGC \\
\hline GAPDH & AAGGGCCCTGACAACTCTTT & CTCCCCTCTTCAAGGGGTCT \\
\hline
\end{tabular}




\section{Supplemental Methods}

\section{Formation of Cerebral organoid (CO)}

hiPSCs were maintained on iMatrix-511 silk coated culture dish in StemFit Basic02 medium supplemented with bFGF $(10 \mathrm{ng} / \mathrm{mL})$. The embryoid bodies were formed using round-bottomed ultra-low attachment 96well plates for 24 hours by seeding the $1.0 \times 10^{5}$ cells per well with StemFit Basic02 medium supplemented with bFGF $(4 \mathrm{ng} / \mathrm{mL})$ and ROCK inhibitor $(50 \mu \mathrm{M})$. After 24 hours from seeding, the embryoid bodies were formed and the medium was replaced with fresh StemFit Basic02 medium with bFGF (4 ng/mL) and ROCK inhibitor $(50 \mu \mathrm{M})$. After 1 day from embryoid body formation (day 2), the medium was replaced with fresh StemFit Basic02 medium. On day 3, the embryoid bodies were transferred to 24-well ultra-low attachment plates. From day 4 to day 8 , the medium was replaced every other day with DMEM/F-12 supplemented with $\mathrm{N} 2$ supplement ( $1 \% \mathrm{v} / \mathrm{v})$, GlutaMAX supplement (1\% v/v), MEM-NEAA solution $(1 \% \mathrm{v} / \mathrm{v})$, and heparin $(1$ $\mu \mathrm{g} / \mathrm{mL}$ ) for neuroepithelial tissue formation. On day 9 , each neuroepithelial tissue was embedded with Matrigel droplet $(5 \mu \mathrm{l})$ on a sterilized $60 \mathrm{~mm}$ dish with Parafilm sheet and placed in a $37{ }^{\circ} \mathrm{C}$ incubator for $20 \mathrm{~min}$ for full polymerization. After the polymerization of Matrigel droplets, add $5 \mathrm{~mL}$ of 1:1 DMEM/F-12:Neurobasal medium supplemented with N2 supplement $(0.5 \% \mathrm{v} / \mathrm{v})$, B-27 supplement without vitamin A (1\% v/v), GlutMAX supplement (1\% v/v), MEM-NEAA solution $(0.5 \% \mathrm{v} / \mathrm{v})$, insulin $(2.5 \mu \mathrm{g} / \mathrm{mL}), 2$-mercaptoethanol $(3.5 \mu \mathrm{L} / \mathrm{L})$ and add penicillin $(100 \mathrm{U} / \mathrm{mL})$ and streptomycin $(100 \mu \mathrm{g} / \mathrm{mL})$. The matrigel droplets were detached from the parafilm sheet and the medium was replaced every other day. On days 14 , the dish with cerebral tissues was placed on orbital shaker $\left(5 \% \mathrm{CO}_{2}\right)$ and the medium was replaced every 3 days with 1:1 DMEM/F12: Neurobasal medium supplemented with $\mathrm{N} 2$ supplement ( $0.5 \% \mathrm{v} / \mathrm{v}), \mathrm{B}-27$ supplement $(1 \% \mathrm{v} / \mathrm{v})$, GlutaMAX supplement (1\% v/v), MEM-NEAA solution $(0.5 \% \mathrm{v} / \mathrm{v})$, insulin $(2.5 \mu \mathrm{g} / \mathrm{mL}), 2$-mercaptoethanol $(3.5 \mu \mathrm{L} / \mathrm{L})$, penicillin $(100 \mathrm{U} / \mathrm{mL})$, and streptomycin $(100 \mu \mathrm{g} / \mathrm{mL})$. The immunostaining and electrophysiological analysis of cerebral organoids were conducted on days 85-95.

\section{Formation of motor neuron spheroids (MNSs)}

Human NSCs were maintained on laminin-coated tissue culture plate with KnockOut DMEM/F-12 medium with StemPro Neural supplement $(20 \mu 1 / \mathrm{mL})$, bFGF (20 ng/mL), EGF (20 ng/mL), GlutaMAX supplement (2 $\mathrm{mM})$, heparin (6 units $/ \mathrm{mL})$ and ascorbic acid $(200 \mu \mathrm{M})$. Neural spheroids were formed using round-bottomed 
ultra-low attachment 96-well plates by seeding the NSCs $\left(5.0 \times 10^{4}\right.$ cells per well). After 24 hours after cell seeding, the culture medium was replaced with StemPro hESC medium with bFGF ( $8 \mathrm{ng} / \mathrm{mL})$, sonic hedgehog (200 ng/mL), activating A $(10 \mathrm{ng} / \mathrm{mL})$, and retinoic acid $(50 \mu \mathrm{M})$ for motor neuron differentiation. After 20 days of culture, the culture medium was replaced with StemPro hESC medium with BDNF (10 ng/mL), GDNF $(10 \mathrm{ng} / \mathrm{mL})$ for maturation of motor neuron, and cultured for 8 days. The immunostaining, PCR, and electrophysiological analysis of cerebral organoids were conducted on days 35-45.

\section{Fabrication of 3D-engineered nervous system (eBSA)}

The mold of the 3D-engineered chip was designed using 3D MAX and fabricated by Stereolithography (SLA) printer with photocurable resin. The surface of molds was coated with trichloro $(1 \mathrm{H}, 1 \mathrm{H}, 2 \mathrm{H}, 2 \mathrm{H}-$ perfluorooctyl)silane for the easy release of replicate from the mold. To generate the replica of a 3D-engineered chip, polydimethylsiloxane (PDMS) and curing reagents were mixed in a 10:1 weight ratio, poured into the mold, and cured at $70{ }^{\circ} \mathrm{C}$ for at least 8 hours. The two parts of the 3D-engineered chip were detached from the mold and treated with oxygen plasma. The 3D-engineered chip was fabricated by assembling the two parts in contact with the oxygen plasma-treated surface.

As shown in Figure S4, the motor neuron spheroids (MNSs) were placed into the co-culture chamber and the medium reservoir 1 was filled up with the motor neuron culture medium. After then, the cerebral organoid culture medium was filled into medium reservoir 2. After the readjusting the sitting position of MNSs, the cerebral organoid was placed on the top of MNSs in the co-culture chamber. The 3D-engineered nervous system (eBSA) was fabricated by fusion of co-cultured motor neuron spheroids and cerebral organoids in 7 days.

\section{Immunocytochemistry}

The CO, MNSs and eBSA were washed with PBS solution and fixed in $4 \%$ paraformaldehyde overnight, and subsequently dehydrated with ethanol, and then became transparent using xylene for paraffin sectioning. After infiltrating and embedding the $\mathrm{CO}, \mathrm{MNSs}$, and eBSA in a paraffin block, the tissues were sectioned at a thickness of $4 \mu \mathrm{m}$. Following the rehydration process of sectioned tissue, primary antibodies were diluted (SOX2 1:50, TUJ 1:250, FOXG1 1:100, TTR 1:50, PROX1 1:50) and treated 1 hour under room temperature. The immunocytochemistry images were obtained by using a confocal microscope (LSM710, Carl Zeiss, 
Germany).

\section{RT-PCR}

To confirm the differentiation into the motor neuron and mRNA expression of adenosine receptor A1 of the cerebral organoid, reverse transcription polymeric chain reaction (RT-PCR) was performed. A genomic RNA was isolated from samples with TRIzol reagent and reverse transcription step was performed using a SuperScript III kit. RT-PCR was performed with a FlexCycler2 system using AccuPower Taq PCR preMix. The mRNA expression level of GAPDH (a housekeeping gene) was used for normalization of all PCR experiments. The primers used were revealed in Supplementary Table1.

\section{Evaluation of electrophysiological activity}

The spontaneous electrophysiological activity of CO, MNS, and eBSA was recorded with multielectrode array (MEA). MEA recording was operated using the Maestro Edge system from Axion BioSystems. A MEA plate containing 16 electrode arrays was precoated with laminin for 1 hour at RT. The samples (cerebral organoid, motor neuron spheroid, and eBSA) were placed on the electrode array and spontaneous electrophysiological activity was recorded $\left(12.5 \mathrm{kHz}\right.$ for $5 \mathrm{~min}$ at $\left.37^{\circ} \mathrm{C}\right)$ in each culture medium. Using Axion Integrated Studio (AxlS 20.4.21), a threshold of 6 x SD and Butterworth bandpass filter with $200 \mathrm{~Hz}$ to 3,000 Hz cutoff frequency were set as an optimal condition to minimize the false positive signals. The electrodes with an average of $\geq 5$ spikes/min were set active condition of sample. The spike raster plots were analyzed by Neural Metric Tool (Axion BioSystems). The various concentration of caffeine was applied to cerebral organoid, motor neuron spheroid and eBSA and spontaneous neuronal firing were recorded every $30 \mathrm{~min}$ for $5 \mathrm{~min}$. 